\title{
Risco de contaminação das águas de superfície e subterrâneas por agrotóxicos recomendados para a cultura do arroz irrigado
}

\author{
Risk assessment of surface and groundwater contamination by the rice pesticides \\ Luiz Fernando Dias Martini' ${ }^{\mathrm{I}}$ Sergiane Souza Caldas" Catia Marian Bolzan" \\ Angela Da Cas Bundt ${ }^{\mathrm{I}}$ Ednei Gilberto Primel $^{\mathrm{II}}$ Luis Antonio de Avila ${ }^{\mathrm{F}}$
}

\section{RESUMO}

A produção agrícola geralmente está associada ao uso de agrotóxicos. Suas características físico-químicas, condições do solo e do ambiente determinam seu destino, assim como o seu potencial de contaminação dos mananciais hídricos. Com o intuito de prever a contaminação do ambiente por agrotóxicos, critérios e modelos matemáticos são utilizados para avaliar o risco de contaminação das águas subterrâneas e superficiais. Diante do exposto, este trabalho teve por objetivo estimar a probabilidade de contaminação de águas subterrâneas e superficiais por agrotóxicos, baseado nas suas características físico-químicas, com auxílio de modelos e parâmetros matemáticos preexistentes. Para análise do risco de contaminação de águas superficiais, foi utilizado o método de Goss, o qual é dividido em dois grupos: potencial de transporte de agrotóxicos dissolvidos em água e adsorvido ao sedimento. Os critérios utilizados para avaliar o potencial de lixiviação foram os sugeridos pela Agência de Proteção Ambiental dos EUA e pelo Índice de GUS. Os resultados obtidos neste trabalho permitem concluir que o uso de modelos de predição do comportamento de agrotóxicos é útil para se obter uma estimativa do risco de contaminação ambiental, pois alguns resultados de monitoramentos confirmam a tendência do comportamento de certos agrotóxicos no ambiente.

Palavras-chave: propriedades físico-químicas, transporte modelos matemáticos.

\section{ABSTRACT}

Agricultural production is generally associated with the use of pesticides, their physicochemical characteristics, soil conditions and the environment will determine their fate, as well as the potential for contamination of water bodies. In order to predict environmental contamination by pesticides, parameters and mathematical models are utilized to assess the risk of contamination of groundwater and surface water. For this reason, the objective of this study was estimate the probability of contamination of groundwater and surface waters by pesticides based on their physicochemical characteristics with the aid of preexisting mathematical models and parameters. To analyze the risk of surface water contamination, the Goss method was used, which as composed by two groups: pesticide potential transport dissolved in water and adsorbed to the sediment. The parameter used to evaluate the leaching potential were those suggested by the US Environmental Protection Agency (EPA) and the GUS Index. The results obtained allow to conclude that the use of models to predict the behavior of pesticides is useful to obtain an estimate of the risk of environmental contamination, as some monitoring results confirm the tendency of the behavior of certain products into the environment.

Key words: physico-chemical, transport, mathematical models.

\section{INTRODUÇÃO}

A cultura do arroz irrigado no Estado do Rio Grande do Sul apresentou, nos últimos anos, elevação dos níveis de produtividade média devido às melhorias de manejo da lavoura e aumento do uso de agrotóxicos. Esses são utilizados para minimizar a interferência de agentes como plantas daninhas, pragas e doenças com a cultura (MARCHEZAN et al., 2010), a qual, desse modo, poderá melhor expressar seu potencial produtivo. Portanto, nesse sistema de cultivo, há uma elevada demanda de herbicidas, inseticidas e nutrientes,

IDepartamento de Fitossanidade, Faculdade de Agronomia Eliseu de Maciel (FAEM), Universidade Federal de Pelotas (UFPel), 96160-990, Pelotas, RS, Brasil. E-mail: laavilabr@gmail.com.*Autor para correspondência.

IIEscola de Química e Alimentos, Universidade Federal do Rio Grande (FURG), Rio Grande, RS, Brasil. 
bem como o elevado uso de água para irrigação (MACHADO et al., 2006).

Quando introduzidos no ambiente, cerca de

$55 \%$ do total de agrotóxicos aplicados não atingem o alvo, dispersando-se para outros compartimentos ambientais como água, solo e atmosfera (GAVRILESCU, 2005). Após atingirem o solo, vários processos físicos, químicos e biológicos determinam seu comportamento. A dinâmica dos agrotóxicos é governada pelo processo de adsorção, o qual é responsável pela ligação desses às partículas do solo, influenciando na disponibilidade para os demais processos como transformação, degradação e transporte, seja por volatilização, lixiviação e/ou por escoamento superficial (HARPER, 1994).

Considerando os processos de transporte entre compartimentos ambientais, a lixiviação e o escoamento superficial merecem destaque. O escoamento superficial favorece a contaminação das águas superficiais, através do transporte do agrotóxico adsorvido às partículas do solo erodido ou dissolvido em água. A lixiviação dos agrotóxicos através do perfil do solo tende a resultar em contaminação das águas subterrâneas, e, nesse caso, as substâncias químicas são carreadas em solução juntamente com a água que alimenta os aquíferos (SPADOTTO et al., 2004). O transporte de agrotóxicos através do perfil do solo se dá principalmente pelo fluxo preferencial ou via zonas de recarga do aquífero (GAVRILESCU, 2005).

Os recursos hídricos agem como integradores dos processos biogeoquímicos de qualquer região, caracterizando-se como destino final de alguns agrotóxicos, podendo ocorrer contaminação destes mananciais, o que pode causar prejuízos para o ecossistema aquático, pois o extravasamento da água da lavoura de arroz irrigado coincide com a época de reprodução de algumas espécies de peixes (PRIMEL et al., 2005). Além disso, tal contaminação pode prejudicar diretamente a saúde humana, principalmente quando esses recursos são utilizados como fonte de água potável para a população (FERRAZ, 1996).

Alguns trabalhos de monitoramento ressaltam a contaminação de águas superficiais, em áreas adjacentes às lavouras de arroz irrigado. Em estudo realizado por BATTAGLIN et al. (2000), em áreas de sequeiro nos EUA, do total de 130 amostras de águas superficiais analisadas, $108(83 \%)$ continham pelo menos um herbicida pertencente ao grupo das sulfonilureias, sulfonamidas e imidazolinonas; destes, destaca-se a presença do herbicida imazetapir em $72 \%$ das amostras, demonstrando a alta capacidade de transporte desse produto amplamente utilizado na lavoura arrozeira gaúcha. Também foi detectada grande frequência de acetocloro (95\%), alacloro (89\%) e atrazina (99\%).
Em trabalho de monitoramento das águas da bacia hidrográfica dos rios Vacacaí e Vacacaí-Mirim, na região Central do Estado do Rio Grande do Sul, MARCHEZAN et al. (2010) demonstraram que o herbicida clomazona apresenta maior incidência nas amostras, o que pode estar correlacionado com a sua persistência no ambiente e sua elevada solubilidade em água, associado à ocorrência de chuvas que promovem o extravasamento da água da lavoura para os rios. Já no estudo de BORTOLUZZI et al. (2007), que avaliaram águas superficiais oriundas de subbacias hidrográficas na região do município de Agudo, também na região Central, foi detectada a presença dos agrotóxicos imidacloprido, atrazina e clomazona, utilizados em cultivos de fumo e arroz. Na região Sul, entre o período da semeadura e colheita do arroz, foi verificado que a qualidade da água do canal São Gonçalo e do rio Piratini é comprometida pela presença de resíduos de carbofurano, quincloraque, clomazona e fipronil (GRUTZMACHER et al., 2008).

O emprego de ferramentas que auxiliem na avaliação preliminar da possibilidade de contaminação por agrotóxicos das águas superficiais, subsuperficiais e subterrâneas de uma área agrícola pode servir de parâmetro para os estudos de contaminação ambiental (FILIZOLA et al., 2002). Com base nas propriedades físico-químicas dos agrotóxicos, é possível estimar o risco de contaminação dos recursos hídricos, através de alguns modelos simplificados como Groundwater Ubiquity Score ou índice de GUS (GUSTAFSON, 1989), critérios propostos pela Agência de Proteção Ambiental dos Estados Unidos (Envinronmental Protection Agency - EPA) (COHEN et al., 1995) e o método de Goss (GOSS, 1992).

Em vista do exposto, este trabalho teve por objetivo estimar a probabilidade de contaminação de águas subterrâneas e superficiais por agrotóxicos recomendados para a cultura do arroz irrigado, baseado em suas características físico-químicas.

\section{MATERIAL E MÉTODOS}

Para análise do risco de contaminação de águas superficiais, foi utilizado o método de Goss (GOSS, 1992), o qual reúne um conjunto de critérios (Tabela 1), em que é considerada a meia vida do herbicida no solo $\left(t_{1 / 2}\right)$, o coeficiente de adsorção ao carbono orgânico $\left(\mathrm{K}_{\mathrm{oc}}\right)$ e a solubilidade em água $\left(\mathrm{S}_{\mathrm{w}}\right)$. Segundo esse método, o transporte de agrotóxicos pode ser separado em dois grupos: transporte de agrotóxicos dissolvidos em água e transporte adsorvido ao sedimento, sendo classificados como de alto, médio e baixo potencial. O agrotóxico é 
Tabela 1 - Critérios utilizados para avaliação do potencial de contaminação de águas superficiais (adaptado de GOSS, 1992).

\begin{tabular}{ccc}
\hline $\mathrm{t}_{1 / 2}$ solo $($ dias $)$ & $\mathrm{K}_{\mathrm{oc}}\left(\mathrm{mL} \mathrm{g}^{-1}\right)$ & Solub. em água $\left(\mu \mathrm{g} \mathrm{mL}^{-1}\right)$ \\
\hline----- Alto potencial de transporte associado ao sedimento------ \\
$=40$ & $=1000$ & ---- \\
$=40$ & $=500$ & $=0,5$ \\
---- -Baixo potencial de transporte associado ao sedimento----- \\
$=1$ & ---- & ---- \\
$=2$ & $=500$ & ---- \\
$=4$ & $=900$ & $=0,5$ \\
$=40$ & $=500$ & $=0,5$ \\
$=40$ & $=900$ & $=2,0$ \\
----- Alto potencial de transporte dissolvido em água-------- & $=1$ \\
$>35$ & $<100.000$ & $10=$ solubilidade $=100$ \\
---- & $=700$ & ---- \\
$------B a i x o$ & potencial de transporte dissolvido em água-------- \\
$=1$ & $=1000$ & $<0,5$ \\
$<35$ & ---- & ---- \\
---- & $=100.000$ &
\end{tabular}

considerado como de médio potencial, quando as propriedades físico-químicas não se enquadram nos parâmetros do método.

Os critérios utilizados para avaliar o potencial de lixiviação (contaminação da água subterrânea) foram os sugeridos pela EPA-EUA (COHEN et al., 1995) e o Índice de GUS (GUSTAFSON, 1989). Os critérios da EPA envolvem os valores de solubilidade em água a $25^{\circ} \mathrm{C}>30 \mathrm{mg} \mathrm{L}^{-1} ; \mathrm{K}_{\mathrm{oc}}<300-500 \mathrm{~cm}^{3}$ $\mathrm{g}^{-1}$; constante da Lei de Henry $\left(\mathrm{K}_{\mathrm{H}}\right)<10^{-2} \mathrm{~Pa} \mathrm{~m}^{3} \mathrm{~mol}^{-1}$, especiação (presença de forma aniônica em $\mathrm{pH}$ normal, entre 5,0 e 8,0 ) e $t_{1 / 2}$ em solo $>21$ dias e na água $>175$ dias; condições de campo que favorecem a percolação no solo, como índice de pluviosidade anual $>250 \mathrm{~mm}$; aquífero não confinado e solo poroso. Agrotóxicos que se enquadram nessas características são considerados potencialmente poluidores de águas subterrâneas.

$O$ índice GUS foi calculado através da equação: $\quad$ GUS $=\log \left(t_{1 / 2}\right){ }^{*}[4-\log (K o c)]$, em que, $t_{1 / 2}$ corresponde à meia vida em campo e $\mathrm{K}_{\mathrm{oc}} \mathrm{o}$ coeficiente de partição com o carbono orgânico do solo. O resultado desse cálculo possibilita classificar os compostos em três categorias, aqueles que não sofrem lixiviação (IL) GUS $<1,8$; compostos que se encontram na faixa de transição (TL) $1,8<$ GUS $<2,8$ e compostos provavelmente lixiviados (PL) GUS $>2,8$.

Os agrotóxicos em estudo são atualmente recomendados para a cultura do arroz irrigado (SOSBAI, 2007) (Tabelas 2 e 3). Suas propriedades físico-químicas foram obtidas do The Pesticide Manual (TOMLIN, 2003); da AGÊNCIA NACIONAL DE VIGILÂNCIA
SANITÁRIA (2009) e do FOOTPRINT (2009). Os dados referentes aos herbicidas foram confirmados com a consulta ao Herbicide Handbook (SENSEMAN, 2007).

\section{RESULTADOS E DISCUSSÃO}

Na tabela 2, são apresentados os resultados para a previsão da contaminação de águas de superfície pelos agrotóxicos utilizando o método de Goss. Na análise de risco de transporte por escoamento superficial associado ao sedimento, os herbicidas bentazona, bispiribaque-sódico, clomazona, cialofopebutílico, 2,4-D, etoxisulfurom, imazapique, pirazossulfurom-etílico, propanil e tiobencarbe; os inseticidas carbaril, fenitrotiona, malationa triclorfom; e os fungicidas azoxistrobina e tiofanato-metílico apresentam baixo potencial. Os herbicidas fenoxapropep-etílico, imazetapir, metsulfurom-metílico, oxifluorfem, pendimetalina, penoxsulam, quincloraque; os inseticidas benfuracarbe, beta-ciflutrina, fipronil, imidacloprido, tiametoxam; e os fungicidas propiconazol e trifloxistrobina são classificados como de médio potencial. Os ingredientes ativos enquadrados como alto potencial adsorvido ao sedimento foram apenas o herbicida glifosato e o inseticida carbofurano.

Em relação à análise de transporte de agrotóxicos dissolvidos em água, os herbicidas clomazona, fenoxaprope-p-etílico, glifosato, imazetapir, imazapique, metsulfurom-metílico, os inseticidas fipronil, imidacloprido, tiametoxam e o fungicida propiconazol apresentam alto potencial de transporte. Esses índices são confirmados em diversos trabalhos de monitoramento de águas de superfície, em que foram encontrados os herbicidas clomazona, imazetapir, imazapique e o inseticida fipronil nos rios Vacacaí e Vacacaí-Mirim, na região central do Estado do Rio Grande do Sul (MARCHEZAN et al., 2010); o inseticida imidacloprido e o herbicida clomazona, na água de enxurrada, na região de Agudo/RS (BORTOLUZZI et al., 2007). Analisando amostras de água coletadas em mananciais hídricos adjacentes a lavouras arrozeiras no RS, SILVA et al. (2009) detectaram pelo menos um agrotóxico em cada amostra, destacando-se o fipronil, seguido de imazetapir, clomazona, tebuconazol, imazapique, quincloraque, penoxsulam e 3-hidróxicarbofurano. A estimativa de contaminação de águas superficiais e subterrâneas também foi efetuada por CABRERA et al. (2008), que verificaram que os agrotóxicos atrazina, carbofurano, clomazona, linurom, metsulfurom-metílico, molinato glifosato possuem alto potencial de contaminação desses dois compartimentos. 
Tabela 2 - Estimativa de contaminação de águas de superfície por herbicidas inseticidas e fungicidas, utilizando os critérios de GOSS (1992).

\begin{tabular}{|c|c|c|}
\hline Nome químico & Potencial de transporte adsorvido no sedimento & Potencial de transporte dissolvido na água \\
\hline \multicolumn{3}{|c|}{ 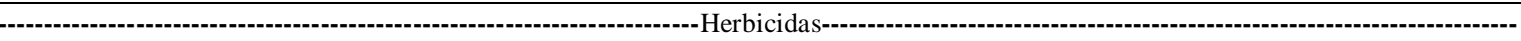 } \\
\hline Bentazona & Baixo & Médio \\
\hline Bispiribaque-sódico & Baixo & Médio \\
\hline Clomazona & Baixo & Alto \\
\hline Cialofope-butílico & Baixo & Baixo \\
\hline $2,4-\mathrm{D}$ & Baixo & Médio \\
\hline Etoxissulfurom & Baixo & Médio \\
\hline Fenoxaprope-P-etílico & Médio & Alto \\
\hline Glifosato & Alto & Alto \\
\hline Imazetapir & Médio & Alto \\
\hline Imazapique & Baixo & Alto \\
\hline Metsulfurom-metílico & Médio & Alto \\
\hline Oxifluorfem & Médio & Médio \\
\hline Pendimetalina & Médio & Médio \\
\hline Penoxsulam & Médio & Baixo \\
\hline Pirazossulfurom-etílico & Baixo & Médio \\
\hline Propanil & Baixo & Médio \\
\hline Tiobencarbe & Baixo & Médio \\
\hline Quincloraque & Médio & Médio \\
\hline \multicolumn{3}{|c|}{ - } \\
\hline Benfuracarbe & Médio & Médio \\
\hline Beta-ciflutrina & Médio & Baixo \\
\hline Carbaril & Baixo & Médio \\
\hline Carbofurano & Alto & Médio \\
\hline Fenitrotiona & Baixo & Médio \\
\hline Fipronil & Médio & Alto \\
\hline Imidacloprido & Médio & Alto \\
\hline Malationa & Baixo & Médio \\
\hline Tiametoxam & Médio & Alto \\
\hline Triclorfom & Baixo & Médio \\
\hline \multicolumn{3}{|c|}{ - } \\
\hline Azoxistrobina & Baixo & Médio \\
\hline Propiconazol & Médio & Alto \\
\hline Tiofanato-metílico & Baixo & Baixo \\
\hline Trifloxistrobina & Médio & Médio \\
\hline
\end{tabular}

Para os herbicidas cialofope-butílico, penoxsulam; para o inseticida beta-ciflutrina; e para o fungicida tiofanato-metílico, foi verificado baixo potencial de transporte dissolvido em água. $\mathrm{O}$ médio potencial foi verificado para os herbicidas bentazona, bispiribaque-sódico, 2,4-D, etoxissulfurom, oxifluorfem, pendimetalina, pirazossulfurom-etílico, propanil, tiobencarbe e quincloraque; e os inseticidas benfuracarbe, carbaril, carbofurano, fenitrotiona, malationa e triclorfom; e os fungicidas azoxistrobina e trifloxistrobina.

Na tabela 3, são apresentados os resultados da avaliação do risco de contaminação das águas subterrâneas, segundo o método de GUS e os critérios
EPA. Os herbicidas bispiribaque-sódico, clomazona, imazetapir, imazapique, metsulfurom-metílico e quincloraque; os inseticidas carbofurano, imidacloprido, tiametoxam e triclorfom são classificados como alta probabilidade de lixiviação, segundo ambos os critérios. Esses resultados corroboram o estudo de BORTOLUZZI et al. (2007), os quais detectaram resíduos do inseticida imidacloprido, entre outros compostos, em fontes de águas subterrâneas. Com relação a monitoramentos de herbicidas, BATTAGLIN et al. (2000), nos EUA, detectaram o herbicida imazetapir em $16 \%$ do total de 25 amostras de águas subsuperficiais, sendo que esse produto foi detectado com maior frequência, entre os 16 herbicidas avaliados. 
Tabela 3 - Avaliação de risco de poluição na água subterrânea por herbicidas, inseticidas e fungicidas com base nos critérios estabelecidos pela EPA-US e pelo índice de GUS (GUSTAFSON, 1989).

\begin{tabular}{|c|c|c|c|c|c|c|c|}
\hline \multirow{2}{*}{ Nome químico } & \multicolumn{5}{|c|}{ - } & \multirow{2}{*}{$\begin{array}{l}\text { Resultado } \\
\text { EPA }^{2}\end{array}$} & \multirow{2}{*}{$\mathrm{GUS}^{3}$} \\
\hline & $\mathrm{S}$ & $\mathrm{K}_{\mathrm{oc}}$ & $\mathrm{K}_{\mathrm{H}}$ & $\mathrm{t}_{1 / 2}$ Solo & $\mathrm{t}_{1 / 2}$ água & & \\
\hline Bentazona & A & A & A & $\mathrm{N}$ & A & PC & $\mathrm{TL}$ \\
\hline Bispiribaque sódico & A & A & A & $\mathrm{N}$ & A & $\mathrm{PC}$ & PL \\
\hline Clomazona & A & A & A & A & $\mathrm{N}$ & $\mathrm{PC}$ & PL \\
\hline Cialofope-butílico & $\mathrm{N}$ & $\mathrm{N}$ & A & $\mathrm{N}$ & $\mathrm{N}$ & $\mathrm{NC}$ & IL \\
\hline 2,4-D & A & A & A & $\mathrm{N}$ & $\mathrm{N}$ & $\mathrm{PC}$ & $\mathrm{TL}$ \\
\hline Etoxissulfurom & A & A & A & $\mathrm{A}$ & $\mathrm{N}$ & $\mathrm{PC}$ & $\mathrm{TL}$ \\
\hline Fenoxaprope-P-etílico & $\mathrm{N}$ & $\mathrm{N}$ & A & $\mathrm{N}$ & $\mathrm{N}$ & $\mathrm{NC}$ & $\mathrm{IL}$ \\
\hline Glifosato & A & $\mathrm{N}$ & A & A & $\mathrm{N}$ & $\mathrm{PC}$ & IL \\
\hline Imazetapir & A & A & A & A & $\mathrm{N}$ & $\mathrm{PC}$ & PL \\
\hline Imazapique & A & A & -- & A & $\mathrm{N}$ & $\mathrm{PC}$ & PL \\
\hline Metsulfurom-metílico & A & A & A & A & A & $\mathrm{PC}$ & PL \\
\hline Oxifluorfem & $\mathrm{N}$ & $\mathrm{N}$ & A & A & $\mathrm{N}$ & $\mathrm{NC}$ & IL \\
\hline Pendimetalina & $\mathrm{N}$ & $\mathrm{N}$ & $\mathrm{N}$ & $\mathrm{A}$ & $\mathrm{N}$ & $\mathrm{NC}$ & PL \\
\hline Penoxsulam & $\mathrm{N}$ & A & A & A & $\mathrm{N}$ & $\mathrm{PC}$ & $\mathrm{TL}$ \\
\hline Pirazossulfurom-etílico & $\mathrm{N}$ & A & -- & A & $\mathrm{N}$ & I & $\mathrm{TL}$ \\
\hline Propanil & A & A & A & $\mathrm{N}$ & $\mathrm{N}$ & $\mathrm{PC}$ & IL \\
\hline Tiobencarbe & A & $\mathrm{N}$ & A & A & A & $\mathrm{PC}$ & $\mathrm{IL}$ \\
\hline Quincloraque & $\mathrm{N}$ & A & A & A & -- & $\mathrm{PC}$ & PL \\
\hline Benfuracarbe & $\mathrm{N}$ & $\mathrm{N}$ & A & $\mathrm{N}$ & $\mathrm{N}$ & $\mathrm{NC}$ & IL \\
\hline Beta-ciflutrina & $\mathrm{N}$ & $\mathrm{N}$ & A & $\mathrm{N}$ & $\mathrm{N}$ & $\mathrm{NC}$ & IL \\
\hline Carbaril & A & A & A & $\mathrm{N}$ & $\mathrm{N}$ & $\mathrm{PC}$ & $\mathrm{TL}$ \\
\hline Carbofurano & A & A & A & A & $\mathrm{N}$ & $\mathrm{PC}$ & PL \\
\hline Fenitrotiona & $\mathrm{N}$ & $\mathrm{N}$ & A & $\mathrm{N}$ & $\mathrm{N}$ & $\mathrm{NC}$ & IL \\
\hline Fipronil & $\mathrm{N}$ & $\mathrm{N}$ & A & A & $\mathrm{N}$ & $\mathrm{NC}$ & PL \\
\hline Imidacloprido & A & A & A & A & $\mathrm{N}$ & $\mathrm{PC}$ & PL \\
\hline Malationa & $\mathrm{A}$ & A & $\mathrm{N}$ & $\mathrm{N}$ & $\mathrm{N}$ & $\mathrm{NC}$ & $\mathrm{IL}$ \\
\hline Tiametoxam & A & A & A & A & $\mathrm{N}$ & $\mathrm{PC}$ & PL \\
\hline Triclorfom & A & A & A & A & -- & $\mathrm{PC}$ & PL \\
\hline Azoxistrobina & $\mathrm{N}$ & $\mathrm{N}$ & $\begin{array}{c}\text { gicic } \\
\mathrm{A}\end{array}$ & $\mathrm{N}$ & $\mathrm{N}$ & $\mathrm{NC}$ & IL \\
\hline Propiconazol & A & $\mathrm{N}$ & A & A & $\mathrm{N}$ & $\mathrm{PC}$ & $\mathrm{TL}$ \\
\hline Tiofanato-metílico & $\mathrm{N}$ & A & A & $\mathrm{N}$ & $\mathrm{N}$ & $\mathrm{NC}$ & IL \\
\hline Trifloxistrobina & $\mathrm{A}$ & $\mathrm{N}$ & A & $\mathrm{N}$ & $\mathrm{N}$ & $\mathrm{NC}$ & IL \\
\hline
\end{tabular}

${ }^{1} \mathrm{~A}=$ atende ao critério com potencial perigoso, $\mathrm{N}=$ não atende ao critério $\mathrm{EPA}$.

${ }^{2} \mathrm{PC}=$ contaminante em potencial, $\mathrm{NC}=$ não contaminante.

${ }^{3} \mathrm{IL}=$ não sofre lixiviação, $\mathrm{TL}=$ faixa de transição, $\mathrm{PL}=$ provável lixiviação.

-- Dados não disponíveis.

No estudo de JOURDAN et al. (1998), foi monitorada a movimentação de imazetapir no perfil de um solo arenoso, o qual, em cinco dias após a aplicação, sob condições moderadas de chuva, foi detectado, nas profundidades de $15-20 \mathrm{~cm}$, podendo atingir até $30 \mathrm{~cm}$ de profundidade após três meses de sua aplicação. Resultados semelhantes foram encontrados por KRAEMER et al. (2009), avaliando a lixiviação de imazetapir em solos de várzea, o qual foi detectado até
$20 \mathrm{~cm}$ de profundidade independente do manejo dado ao solo, plantio convencional ou direto, sendo observadas maiores concentrações na camada de solo de $5-15 \mathrm{~cm}$, no sistema de plantio direto. Os herbicidas pertencentes ao grupo químico das imidazolinonas, como o imazetapir e imazapique, possuem grande persistência e alta solubilidade em água, conferindolhes maior probabilidade de contaminação de águas subterrâneas, devido à sua grande mobilidade no solo 
(JOURDAN et al., 1998; FIRMINO et al., 2008). Os herbicidas cialofope-butílico, fenoxaprope-p-etílico e oxifluorfem; os inseticidas benfuracarbe, beta-ciflutrina, fenitrotiona e malationa; e os fungicidas azoxistrobina, tiofanato-metíllico e trifloxistrobina não apresentam tendência de lixiviação, para ambas as avaliações.

Comparando-se os critérios do EPA com o critério do GUS, alguns agrotóxicos diferem na sua classificação. Os herbicidas glifosato, propanil e tiobencarbe são classificados como não lixiviáves pelo método de GUS, porém considerados como potenciais contaminantes de águas subsuperficiais pelos critérios EPA. Já para o herbicida pendimetalina e para o inseticida fipronil, ocorre o contrário, já que são classificados com provável lixiviação pelo método de GUS e não contaminantes pelos critérios EPA. Os herbicidas bentazona, 2,4-D, etoxissulfurom e penoxsulam, o inseticida carbaril e o fungicida propiconazol passaram da faixa de transição na avaliação de GUS para contaminante em potencial nos critérios EPA. Pirazossulfurom-etílico não obteve resultado conclusivo por EPA, devido à carência de informações sobre o ingrediente ativo.

O uso de modelos de predição do comportamento de agrotóxicos é útil para se obter uma estimativa do risco de contaminação ambiental e auxilia na escolha dos agrotóxicos a serem monitorados nos corpos hídricos, pois alguns resultados de monitoramentos confirmam a tendência do comportamento de certos compostos no ambiente.

\section{CONCLUSÃO}

Com relação ao risco de contaminação de águas superficiais, o herbicida glifosato e o inseticida carbofurano apresentam alto potencial de transporte por escoamento superficial adsorvido ao sedimento. $\mathrm{Na}$ avaliação de transporte dissolvido em água, os herbicidas clomazona, fenoxaprope-P-etílico, glifosato, imazetapir, imazapique e metsulfurom-metílico; os inseticidas fipronil, imidacloprido e tiametoxam; e o fungicida propiconazol apresentam alto potencial.

A respeito do risco de contaminação de águas subterrâneas, usando-se os critérios do EPA, os herbicidas que apresentam potencial contaminador são: bentazona, bispiribaque-sódico, clomazona, 2,4-D, etoxisulfurom, glifosato, imazetapir, imazapique, metsulfurom-metílico, penoxsulam, propanil, tiobencarbe e quincloraque; os inseticidas carbaril, carbofurano, imidacloprido, tiametoxam e triclorfom; $\mathrm{e}$ o fungicida propiconazol. Já, usando-se o método de GUS, os herbicidas que apresentam maior probabilidade de lixiviação para águas subterrâneas são: bispiribaque- sódico, clomazona, imazetapir, imazapique, metsulfurom-metílico, pendimetalina e quincloraque; os inseticidas carbofurano, fipronil, imidacloprido, tiametoxam e triclorfom.

\section{REFERÊNCIAS}

BATTAGLIN, W.A. et al. Occurrence of sulfonylurea, sulphonamide, imidazolinone and other herbicides in rivers, reservoirs and ground water in the Midwestern United States, 1998. Science Total Environmental, v.248, n.2-3, p.123133. 2000. Disponível em: <http://co.water.usgs.gov/ midconherb/pdf/battaglin99.pdf>. Acesso em: 05 jun. 2010. doi: 10.1016/S0048-9697(99)00536-7.

BORTOLUZZI, E.C. et al. Investigation of the occurrence of pesticides residues in rural wells and surface water following application to tobacco. Química Nova, v.30, n.8, p.1872-1876, 2007. Disponível em: <http://www.scielo.br/scielo.php?pid=S010040422007000800014\&script $=$ sci_arttext $>$. Acesso em: 05 jun. 2010. doi: 10.1590/S0100-40422007000800014.

BRASIL. Agência Nacional de Vigilância Sanitária. Sistema de Informações Sobre Agrotóxicos, 2009. Disponível em: <http:/ /www4.anvisa.gov.br/agrosia/asp/frm_dados_ingrediente.asp>. Acesso em: 03 jun. 2010.

CABRERA, L.C. et al. Estimativa de risco de contaminação das águas por pesticidas na região sul do estado do RS. Química Nova, v.31, p.1982-1986, 2008. Disponível em: <http://www.scielo.br/ scielo.php?pid=S0100-40422008000800012\&script $=$ sci_arttext $>$. Acesso em: 08 jun. 2010. doi: 10.1590/S010040422008000800012 .

COHEn, S.Z. et al. Pure and Applied Chemistry, v.67, p.2109-2148, 1995. Disponível em: <http://www.iupac.org/ publications/pac/1995/pdf/6712x2109.pdf>. Acesso em: 03 jun. 2010 .

FERRAZ, E.S.B. A bacia hidrográfica como unidade de estudo, estrutura e processos (apresentação). In: WORKSHOP DO PROJETO PIRACENA, 2., 1966, Piracicaba, SP. Anais... Piracicaba:CENA, 1996. p.7.

FILIZOLA, H.F. et al. Monitoramento e avaliação do risco de contaminação por pesticidas em água superficial e subterrânea na região de Guaira. Pesquisa Agropecuária Brasileira, v.37, n.5, p. 659-667, 2002. Disponível em: <http:// www.scielo.br/pdf/pab/v37n5/9535.pdf >. Acesso em: 08 jun. 2010. doi: 10.1590/S0100-204X2002000500011.

FIRMINO, L.E. et al. Movimento do herbicida imazapyr no perfil de solos tropicais. Planta Daninha, v.26, n.1, p.223-230, 2008. Disponível em: <http://www.scielo.br/scielo.php?pid=S0100$83582008000100023 \&$ script=sci_arttext $>$. Acesso em: 08 jun. 2010. doi: 10.1590/S0100-83582008000100023.

FOOTPRINT: creating tools for pesticide risk assessment and management in Europe, 2009. Disponível em: <http://www.eufootprint.org/>. Acesso em: 03 jun. 2010.

GAVRILESCU, M. Fate of pesticides in the environment and its bioremediation. Engineering in Life Sciences, v.5, n.6, p.497526, 2005. Disponível em: <http://onlinelibrary.wiley.com/doi/ 
10.1002/elsc.200520098/pdf>. Acesso em: 08 jun. 2010. doi: $10.1002 /$ elsc. 200520098 .

GOSS, D.W. Screening procedure for soils and pesticides for potential water quality impacts. Weed Technology, v.6, n.4, p.701-708, 1992. Disponível em: <http://www.jstor.org/pss/ 3987238>. Acesso em: 08 jun. 2010.

GRUTZMACHER, D.D. et al. Monitoramento de agrotóxicos em dois mananciais hídricos no sul do Brasil. Revista Brasileira de Engenharia Agrícola e Ambiental, v.12, n.6, p.632637, 2008. Disponível em: <http://www.scielo.br/pdf/rbeaa/ v12n6/v12n06a10.pdf>. Acesso em: 08 jun. 2010. doi: $10.1590 / \mathrm{S} 1415-43662008000600010$.

GUSTAFSON, D.I. Groundwater ubiquity score: a simple method for assessing pesticide leachability. Environmental Toxicology and Chemistry, v.8, p.339-357, 1989. Disponível em: <http:/ /onlinelibrary.wiley.com/doi/10.1002/etc.5620080411/pdf>. Acesso em: 03 jun. 2010. doi: 10.1002/etc.5620080411.

HARPER, S. Sorption-desorption and herbicide behavior in soil. Weed Science, v.6, p.207-225, 1994.

JOURDAN, S.W. et al. Imazethapyr bioactivity and movement in soil. Weed Science, v.46, p.608-613, 1998. Disponível em: <http://www.jstor.org/pss/4045969>. Acesso em: 08 jun. 2010.

KRAEMER, A.F. et al. Lixiviação do imazethapyr em solo de várzea sob dois sistemas de manejo. Ciência Rural, v.39, n.6, p.1660-1666, 2009. Disponível em: <http://www.scielo.br/ scielo.php?pid $=$ S0100-83582009000300020\&script $=$ sci_arttext $>$. Acesso em: 10 jun. 2010. doi: 10.1590/S010083582009000300020 .

MACHADO, S.L.O. et al. Consumo de água e perdas de nutrientes e de sedimentos na água de drenagem inicial de arroz irrigado. Ciência Rural, v.36, n.1, p.65-71, 2006. Disponível em: 〈http://www.scielo.br/pdf/cr/v36n1/a10v36n1.pdf>. Acesso em: 10 jun. 2010. doi: 10.1590/S0103-84782006000100010.

MARCHEZAN, E. et al. Resíduos de agrotóxicos na água de rios da Depressão Central do Estado do Rio Grande do Sul, Brasil. Ciência Rural, v.40, p.1053-1059, 2010. Disponível em: <http://www.scielo.br/pdf/cr/v40n5/a574cr2775.pdf>. Acesso em: 10 fev. 2011. doi: 10.1590/S010384782010005000078 .

PRIMEL, E.G. et al. Poluição das águas por herbicidas utilizados no cultivo do arroz irrigado na região central do estado do Rio Grande Do Sul, Brasil: predição teórica e monitoramento. Química Nova, v.28, n.4, p.605-609, 2005. Disponível em: < http://www.scielo.br/scielo.php? pid=S 0100 40422005000400010\&script=sci_arttext $>$. Acesso em: 10 jun. 2010. doi: 10.1590/S0100-40422005000400010.

SENSEMAN, S. Herbicide handbook. USA: Weed Science Society of America, 2007. 459p.

SILVA, D.R.O. et al. Monitoramento de agrotóxicos em águas superficiais de regiões orizícolas no sul do Brasil. Ciência Rural, v.39, p.2383-2389, 2009.

SOCIEDADE SUL-BRASILEIRA DE ARROZ IRRIGADO (SOSBAI). Arroz irrigado: recomendações técnicas da pesquisa para o Sul do Brasil. Pelotas, RS: SOSBAI, 2007. 161p.

SPADOTTO, C.A. et al. Monitoramento do risco ambiental de agrotóxicos: princípios e recomendações. Jaguariúna: Embrapa Meio Ambiente, 2004. 29p. (Documentos 42).

TOMLIN, C.D.S. The pesticide manual. 13.ed. Londres: British Crop Protection Council, 2003. 1350p. 